\title{
Universal trend of the information entropy of a fermion in a mean field
}

\author{
C.P. Panos, S.E. Massen and C.G. Koutroulos \\ Department of Theoretical Physics, Aristotle University of Thessaloniki, 54006 Thessaloniki, Greece
}

\begin{abstract}
We calculate the information entropy of single-particle states in position-space $S_{r}$ and momentumspace $S_{k}$ for a nucleon in a nucleus, a $\Lambda$ particle in a hypernucleus and an electron in an atomic cluster. It is seen that $S_{r}$ and $S_{k}$ obey the same approximate functional form as functions of the number of particles, $S_{r}$ (or $S_{k}$ ) $=a+b N^{1 / 3}$ in all of the above many-body systems in positionand momentum- space separately. The net information content $S_{r}+S_{k}$ is a slowly varying function of $N$ of the same form as above. The entropy sum $S_{r}+S_{k}$ is invariant to uniform scaling of coordinates and a characteristic of the single-particle states of a specific system. The order of single-particle states according to $S_{r}+S_{k}$ is the same as their classification according to energy keeping the quantum number $n$ constant. The spin-orbit splitting is reproduced correctly. It is also seen that $S_{r}+S_{k}$ enhances with excitation of a fermion in a quantum-mechanical system. Finally, we establish a relationship of $S_{r}+S_{k}$ with the energy of the corresponding single-particle state i.e. $S_{r}+S_{k}=k \ln (\mu E+\nu)$. This relation holds for all the systems under consideration.

PACS: 89.70.+c; 36.40.+d; 31.10.+z; 21.60.-n
\end{abstract}

\section{INTRODUCTION}

The information entropy for a continuous probability distribution $p(x)$ in one dimension is defined by the expression:

$$
S=-\int p(x) \ln (p(x)) d x
$$

where $\int p(x) d x=1$.

$S$ is measured in bits if the base of the logarithm is 2 and nats (natural units of information) if the logarithm is natural. It represents the information content of a probability distribution as well as a measure of uncertainty of the corresponding state. We note that the information and thermodynamic entropy are different concepts but can be connected employing some assumptions.

An important step in the past was the discovery in [1] of an entropic uncertainty relation (EUR), which for a three-dimensional system has the form:

$$
S_{r}+S_{k} \geq 3(1+\ln \pi) \cong 6.434, \quad(\hbar=1)
$$

where

$$
S_{r}=-\int \rho(\vec{r}) \ln \rho(\vec{r}) d \vec{r}
$$

is the information entropy in position-space

$$
S_{k}=-\int n(\vec{k}) \ln n(\vec{k}) d \vec{k}
$$

is the information entropy in momentum-space and $\rho(\vec{r}), n(\vec{k})$ are the density distributions in position- and momentumspace respectively, normalized to unity.

The lower bound in (2) is attained for gaussian density distributions. The physical meaning of the above inequality is the following: an increase of $S_{k}$ corresponds to a decrease of $S_{r}$ and vice versa, which indicates that a diffuse density distribution $n(k)$ in momentum space is associated with a localised density distribution $\rho(r)$ in configuration space and vice versa.

Relation (2) represents a strengthened version of Heisenberg's uncertainty principle for two reasons: first EUR leads to Heisenberg's uncertainty relation but the inverse is not true. Second the right-hand-side of EUR does not depend on the state of the system, while in Heisenberg's relation does depend. It is obvious from (3) and (4) that $S_{r}, S_{k}$ depend on the unit of length in measuring $\rho(\vec{r})$ and $n(\vec{k})$. However, the important quantity is the entropy sum $S_{r}+S_{k}$ (net information content of the state) which is invariant to uniform scaling of coordinates. 
Information entropy was employed in the past for the study of quantum mechanical systems [1-9]. Recently [10] we studied the position- and momentum- space information entropies $S_{r}, S_{k}$ respectively for various systems: the nuclear density distribution of nuclei, the electron density distribution of atoms and the valence electron density distribution of atomic clusters. We showed that a similar functional form $S=a+b \ln N$ for the entropy as function of the number of particles $N$ holds approximately for the above systems. We conjectured that this is a universal property of a many-fermion system in a mean field.

The concept of information entropy proved also to be fruitful in a different context [9]. We used the formalism of Ghosh, Berkowitz and Parr 111] within the ground state density functional framework, to define the concept of an information entropy associated with the density distribution of a nuclear system. It turned out that $S$ increases with the quality of the wave function and can serve as a criterion of the quality of a nuclear model.

Another interesting result [12] is the fact that the entropy of an $N$-photon state subjected to Gaussian noise increases linearly with the logarithm of $N$.

Encouraged by previous work we attempt in the present paper to calculate $S_{r}, S_{k}$ for the wave functions of singleparticle states (instead of the total densities as in [10]) for various systems i.e. a nucleon in a nucleus, an electron in an atomic cluster and a $\Lambda$ particle in a hypernucleus. We employ for these systems models existing in the literature.

Our aim is to investigate the dependence of $S_{r}, S_{k}$ on the excitation of a fermion in a quantum-mechanical system as well as its dependence on the system under consideration and the number of the particles $N$. We also attempt to connect the information entropy with the energy of the single-particle state. The study of the dependence of $S$ on the quantum state of a system is also interesting (as stated in [7]) for two reasons: (i) The information-theoretical and physical entropy are connected via Boltzmann's constant $k_{B}$ by the Jayne's relation $S_{p h y s}=k_{B} S_{i n f}$. Thus one can ascribe to any quantum object a certain value of its physical entropy $S_{\text {phys }}$ if one calculates $S_{\text {inf }}$. (ii) It is interesting to know the value of the information entropy which is a measure of the spatial "spreading out" of the wave function for various states of various systems.

The present paper is organized as follows: In sec. 2 we calculate $S_{r}, S_{k}$ for single-particle states of a nucleon in a nucleus as function of the number of nucleons $N$ using the simplest model available i.e. the harmonic oscillator potential and a more realistic one (Skyrme). In sec. 3 we calculate $S_{r}, S_{k}$ for a $\Lambda$ particle in a hypernucleus employing a simple and (semi-) analytical relativistic model. In sec 4 we determine $S_{r}, S_{k}$ for the single-particle states of an electron in atomic (metallic) clusters using the Woods-Saxon potential. In sec. 5 we present a relationship of $S_{r}+S_{k}$ with the energy. Finally, sec. 6 contains a discussion of our results (comparison of sec. 2, 3 and 4) and our conclusions.

\section{INFORMATION ENTROPY FOR A NUCLEON IN A NUCLEUS}

The information entropy $S_{r}$ in position-space for a single-particle wave function $\psi(\vec{r})$ is defined as

$$
S_{r}=-\int|\psi(\vec{r})|^{2} \ln |\psi(\vec{r})|^{2} d \vec{r}
$$

while the entropy $S_{k}$ in momentum-space is

$$
S_{k}=-\int|\phi(\vec{k})|^{2} \ln |\phi(\vec{k})|^{2} d \vec{k}
$$

where $\phi(\vec{k})$ is the Fourier transform of $\psi(\vec{r})$.

In this section we calculate $S_{r}$ and $S_{k}$ for the single-particle states $1 s, 1 p, 1 d, \cdots$ of a nucleon in a nucleus in the framework of the harmonic oscillator ( $\mathrm{HO}$ ) model. We use for the HO parameter the well-known expression $\hbar \omega=41 A^{-1 / 3} \mathrm{MeV}$.

We find that the value of $\hbar \omega$ is important only for $S_{r}, S_{k}$, while the net information content $S=S_{r}+S_{k}$ is independent of $\hbar \omega$ and consequently of $A$. It depends only on the state under consideration and characterises it. These values for the states $1 s, 1 p, 1 d$ and $2 s$ are $6.4341,7.8388,8.6651$ and 8.3015 respectively.

However, the HO model is a simplification. Thus, we employed a more realistic parametrization of the nuclear mean field i.e. the Skyrme (Sk III) interaction [13]. In this model protons and neutrons move in different potentials. We choose to work with protons. However, similar results can be obtained for neutrons. We found that the values for $S_{r}, S_{k}$ obtained from the wave functions of single-particle states calculated according to Sk III are represented well by the expression

$$
S_{r}\left(\text { or } S_{k}\right)=a+b N^{1 / 3}
$$

while $S_{r}+S_{k}$ is a slowly varying function of $N$ of the same form as (7). The values of the parameters are shown in Table 1. 
In Fig. 1a we plot our fitted expressions (Sk III) $S_{r}\left(\right.$ or $\left.S_{k}\right)=a+b N^{1 / 3}$ for the entropies $S_{r}, S_{k}, S_{r}+S_{k}$ of $1 s$-states as functions of $N^{1 / 3}$. The lines correspond to our fitted expressions, while the corresponding values of our numerical calculations are denoted by squares for $S_{r}$, circles for $S_{k}$ and triangles for $S_{r}+S_{k}$. Similar graphs can be plotted for the higher states $1 p, 1 d, 2 s, \cdots$. From Fig. 1a we see that the values of the entropies are represented well by our fitted expressions. In Fig. 1b we compare the sum $S_{r}+S_{k}=a+b N^{1 / 3}$ for various single-particle states. We observe that the entropy sum $S_{r}+S_{k}$ enhances with the excitation of the single-particle states. We see that $S_{r}+S_{k}$ is a slowly

varying function of $N$. We also note that the spin-orbit splitting is reproduced correctly i.e. the state $1 p_{3 / 2}$ is lower than $1 p_{1 / 2}$ e.t.c. (as for the energy) although their difference is small and cannot be shown in the figure.

\section{INFORMATION ENTROPY FOR A $\Lambda$ IN A HYPERNUCLEUS}

We employ a simple and (semi-) analytical relativistic model of a hypernucleus of ref. [14], 15], where the Dirac equation with a scalar potential $U_{S}(r)$ and the fourth component of a vector potential $U_{V}(r)$ was considered in the case of rectangular shapes of these potentials with the same radius:

$$
R=r_{0} A_{\text {core }}^{1 / 3}
$$

In [14 the Dirac equation was solved and gave the wave functions $G(r)$ and $F(r)$ for the large and small components for a $\Lambda$ particle in a hypernucleus. These components can be found in relations (10) and (11) of [14.

The Dirac spinors in terms of large $(G)$ and small $(F)$ components can be expressed:

$$
\psi=\left(\begin{array}{c}
i G(r) / r \\
F(r) / r
\end{array}\right)
$$

The density distribution of a $\Lambda$ in position space is:

$$
\rho(r)=\frac{1}{4 \pi}\left[G^{2}(r) / r^{2}+F^{2}(r) / r^{2}\right]
$$

and the normalization is

$$
4 \pi \int_{0}^{\infty} \rho(r) r^{2} d r=1
$$

In momentum-space we have:

$$
\phi(k)=\left(\begin{array}{c}
i X(k) \\
Y(k)
\end{array}\right)
$$

where $X(k)$ and $Y(k)$ are the Fourier transforms of $G(r) / r$ and $F(r) / r$ respectively. Thus the density distribution in momentum-space is given by:

$$
n(k)=\frac{1}{4 \pi}\left[X^{2}(k)+Y^{2}(k)\right]
$$

and the normalization is:

$$
4 \pi \int_{0}^{\infty} n(k) k^{2} d k=1
$$

The information entropies of the $\Lambda$ particle are calculated according to the relations:

$$
\begin{aligned}
& S_{r}=-4 \pi \int \rho(r) \ln \rho(r) r^{2} d r \\
& S_{k}=-4 \pi \int n(k) \ln n(k) k^{2} d k
\end{aligned}
$$

where $\rho(r)$ and $n(k)$ are given by (9) and (11) respectively. 
For the depths of the potential we used the values 14 : $D_{+}=30.55 \mathrm{MeV}, D_{-}=300 \mathrm{MeV}, r_{0}=1.01 \mathrm{fm}$ and the radius parameter $R=r_{0} A_{\text {core }}^{1 / 3}$ obtained by fitting the experimental binding energies of the ground state of the $\Lambda$ particle. In the following we put $A_{\text {core }}=N=$ number of particles.

Next we fitted the expressions $S_{r}\left(\right.$ or $\left.S_{k}\right)=a+b N^{1 / 3}$ to the values of $S_{r}, S_{k}$ calculated from (12) and (13) and found that these values are represented well. The values of the parameters $a$ and $b$ for various states are shown in Table 1.

In Fig. 2a we plot our fitted expressions for $S_{r}, S_{k}, S_{r}+S_{k}$ as functions of $N^{1 / 3}$ for the $1 s$ state. This is done for a $\Lambda$ in a hypernucleus in a similar way as for a nucleon in a nucleus (Fig 1a). Similar graphs can be plotted for the higher states. In fig. $2 \mathrm{~b}$ we compare the sum $S_{r}+S_{k}$ for various single-particle states of a $\Lambda$ (similar with Fig $1 \mathrm{~b}$ ). The spin orbit splitting is reproduced correctly as in nuclei (Sec. 2).

\section{INFORMATION ENTROPY FOR AN ELECTRON IN AN ATOMIC CLUSTER.}

We consider atomic (metallic) clusters composed of neutral sodium atoms, where the electrons move in an effective radial electronic potential parametrized by Woods-Saxon potential of the form:

$$
V_{W S}(r)=\frac{-V_{0}}{1+\exp [(r-R) / a]}
$$

with $V_{0}=6 \mathrm{eV}, R=r_{0} N^{1 / 3}, r_{0}=2.25 \AA$ and $a=0.74 \AA$. For a detailed study regarding the parametrization of Ekardt's potentials see ref [16].

We found the wave functions of the single-particle states in configuration space and by Fourier transform the corresponding ones in momentum space by solving numerically the Schröndinger equation for atomic clusters for various values of the number of valence electrons $N$. Using the above wave functions, we calculated the information entropies $S_{r}, S_{k}$ (relations (5) and (6) ) for the single-particle states instead of the total density distributions as in ref. [10]. Then we fitted the form $S_{r}\left(\right.$ or $\left.S_{k}\right)=a+b N^{1 / 3}$ to these values and found that these expressions represent well the values of $S_{r}, S_{k}$. In Fig. 3 a we plot $S_{r}, S_{k}$ and $S_{r}+S_{k}$ as functions of $N^{1 / 3}$ (similar as Fig. 1a, 2a) and in Fig. $3 \mathrm{~b}$ we compare $S_{r}+S_{k}$ for various states (similar as in Fig. 1b, 2b). In Table 1 we present the values of the parameters $a$ and $b$ which were obtained from the fitting.

\section{RELATIONSHIP OF THE INFORMATION ENTROPY WITH THE ENERGY OF SINGLE-PARTICLE STATES}

In Fig. 4 we plot $S_{r}+S_{k}$, obtained with the HO model of the nucleus, versus the energy of the single-particle states. We use $\hbar \omega=41 A^{-1 / 3}$ with $A=208(\mathrm{~Pb})$ and keep the quantum number $n$ equal to 1 . A fitting procedure gives for $n=1$, the relation:

$$
S=k \ln (\mu E+\nu)
$$

where $k=2.0206, \mu=3.5373 \mathrm{MeV}^{-1}$ and $\nu=-12.5320$. Similar relations hold for $n>1$.

Next we plot the sum $S_{r}+S_{k}$ as function of the energy $E$ of single-particle states for a proton in a nucleus according to Sk III interaction for ${ }^{208} \mathrm{~Pb}$ (Fig. 5) and an electron in atomic cluster with $N=198$ (Fig. 6) for $n=1$. Similar curves hold for higher values of $n>1$. In both cases the dependence of $S_{r}+S_{k}$ on $E$ can be represented well by the functional form (15). The values of the constants are the following

$$
\begin{aligned}
& k=1.5262, \quad \mu=17.3043 \mathrm{MeV}^{-1}, \quad \nu=793.109 \quad \text { for a proton in a nucleus } \\
& k=1.2386, \quad \mu=1481.48 \mathrm{eV}^{-1}, \quad \nu=8730.52 \quad \text { for an electron in a cluster }
\end{aligned}
$$

A similar relation may be obtained for a $\Lambda$ in a hypernucleus but the number of values of $S_{r}+S_{k}$ available is small. It is the first time in our research on information entropy that we observe such a relationship of $S_{r}+S_{k}$ with a fundamental quantity as the energy. We note that in Fig. 5 there are pairs of points with almost the same $S_{r}+S_{k}$ which reproduce the spin-orbit splitting. 


\section{DISCUSSION AND CONCLUSIONS}

Comparing our results in sections 2,3 , and 4 , we see that a similar functional form $S_{r}\left(\right.$ or $\left.S_{k}\right)=a+b N^{1 / 3}$ describes well the information entropies $S_{r}, S_{k}$ of the single-particle states for a nucleon in a nucleus, a $\Lambda$ in a hypernucleus and a valence electron in an atomic cluster, although the single-particle potentials are different. We conjecture that this is a universal trend of the information entropies $S_{r}, S_{k}$ for a fermion in a mean field, while the net information content $S_{r}+S_{k}$ of the single-particle states of a fermion in a mean field is a slowly varying function of $N$ of the form $S=a+b N^{1 / 3}$ for the systems considered above. For nuclei and the simple HO potential $S_{r}+S_{k}$ is exactly a constant independent of $N$ i.e. $b=0$.

We note that in [10] we found the universal property $S=a+b \ln N$ for the total density distributions of various systems.

In both cases it is not clear why $S$ depends linearly on $\ln N$ (total densities) or linearly on $N^{1 / 3}$ (single-particle states) but we note that in atomic physics there is already a connection of the information entropy with experiment i.e. with fundamental and/or experimental quantities e.g. the kinetic energy or the magnetic susceptibility. Both characteristics have been used in the study of the dynamics of atomic and molecular systems [17]. This connection established the information entropy as an interesting entity for atomic physics. In the present paper we obtained a relationship of $S_{r}+S_{k}$ with a fundamental quantity as the energy of the single-particle states, i.e. $S=k \ln (\mu E+\nu)$. It is remarkable that the same functional form holds for various systems.

[1] I. Bialynicki-Birula, J.Mycielski, Commun. Math. Phys. 44 (1975) 129.

[2] S.R. Gadre, Phys. Rev. A 30 (1984) 620.

[3] S.R. Gadre, S.B. Sears, S.J. Chacravorty, R.D. Bendale, Phys. Rev. A 32 (1985) 2602.

[4] S.R. Garde, R.D. Bendale, Phys. Rev. A 36 (1987) 1932.

[5] M. Ohya, P. Petz, "Quantum entropy and its use" (Springer Berlin, 1993).

[6] A. Nagy, R.G. Parr, Int. J. Quant. Chem. 58 (1996) 323.

[7] V. Majernic, T. Opatrny, J. Phys. A 29 (1996) 2187.

[8] C.P. Panos, S.E. Massen, Int.J. Mod. Phys. E 6 (1997) 497.

[9] G.A. Lalazissis, S.E. Massen, C.P. Panos, S.S. Dimitrova, Int. J. Mod. Phys. E 7 (1998) 485.

[10] S.E. Massen, C.P. Panos, Phys. Lett. A 246 (1998) 530.

[11] S.K. Ghosh, M. Berkowitz, R.G. Parr, Proc. Natl. Acad. Sci. USA 81 (1984) 8028.

[12] M. Hall, Phys. Rev. A 50 (1994) 3295.

[13] C.B. Dover, N.Van Giai, Nucl. Phys. A 190 (1972) 373.

[14] M.E. Grypeos, C.G. Koutroulos and G.J. Papadopoulos, Phys. Rev. A 50 (1994) 29.

[15] G.J. Papadopoulos, C.G. Koutroulos and M.E. Grypeos, Int. J. Theor. Phys. 39 (2000) 455.

[16] B.A.Kotsos, M.E. Grypeos, Physica B 229 (1997) 173.

[17] R.G. Parr, W.Yang, "Density functional theory of atoms and molecules (Oxford Univ. Press, New York, 1989). 

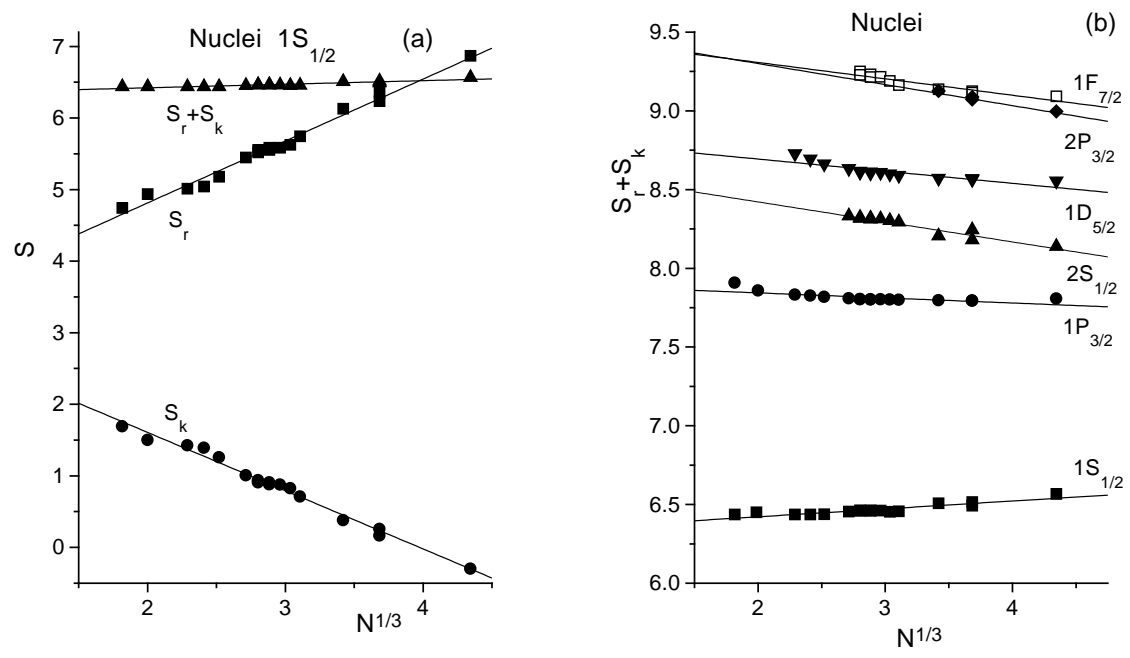

FIG. 1. (a) Values of the information entropies $S_{r}$ (squares), $S_{k}$ (circles) and $S_{r}+S_{k}$ (triangles), calculated numerically, versus the number of particles $N$. These values correspond to the single-particle states of a proton in various nuclei, according to the Sk III interaction. The lines correspond to our fitted expressions $S_{r}$ (or $\left.S_{k}\right)=a+b N^{1 / 3}$. (b) Comparison of the sum $S_{r}+S_{k}$ for various proton single-particle states.
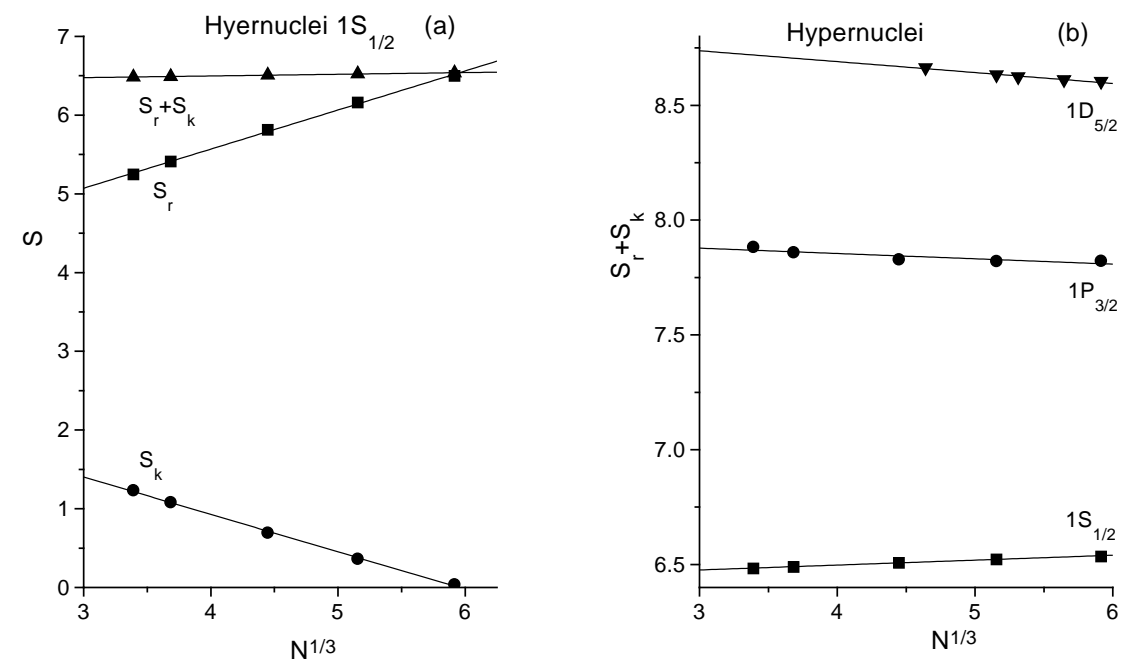

FIG. 2. The same as in Fig. 1 for a $\Lambda$ in hypernuclei employing a relativistic model. 

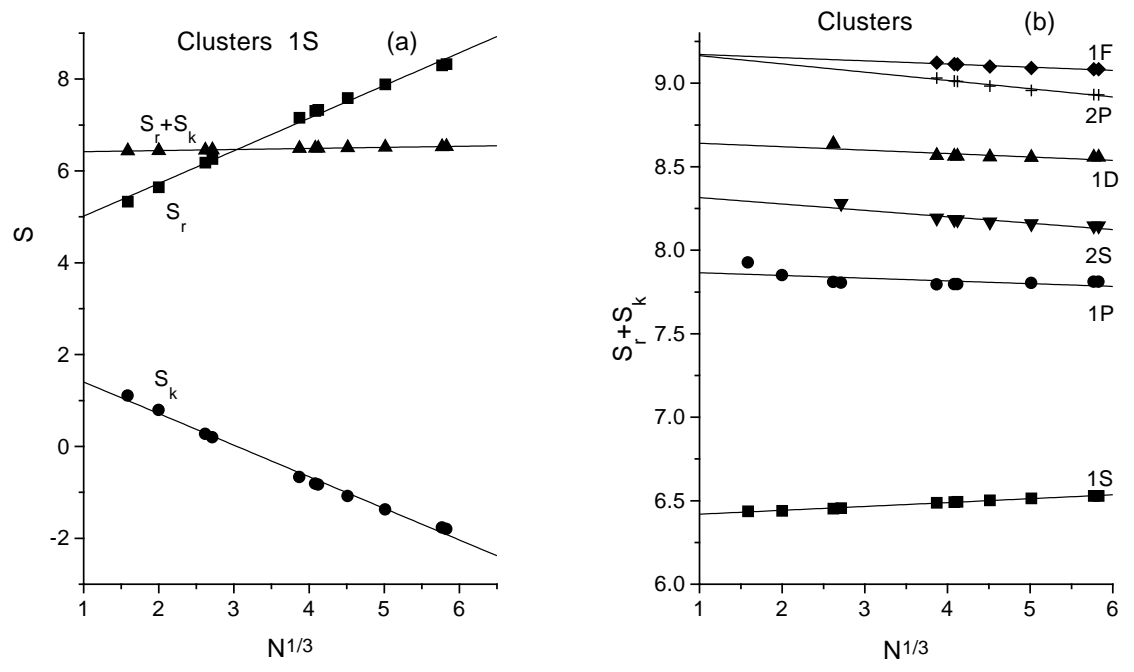

FIG. 3. The same as in Fig. 1 for an electron in atomic clusters with the Woods-Saxon potential.

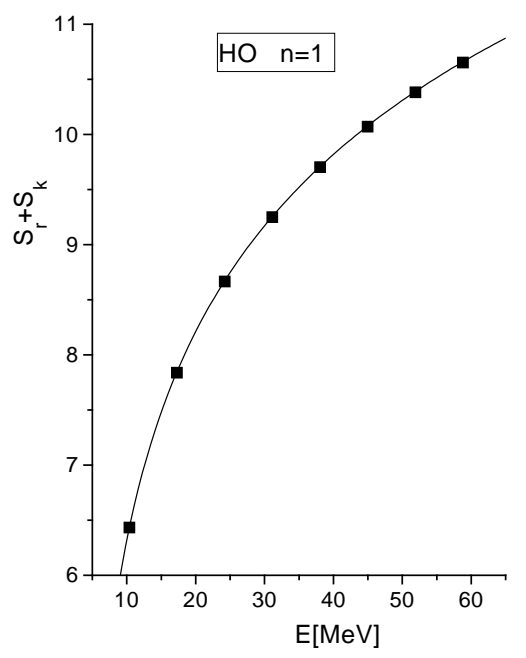

FIG. 4. The values of the energy $E$ (squares) of single-particle states for a nucleon in $\mathrm{Pb}^{208}$ according to the HO model for $n=1$. The line corresponds to our fitted expression $S=k \ln (\mu E+\nu)$. 


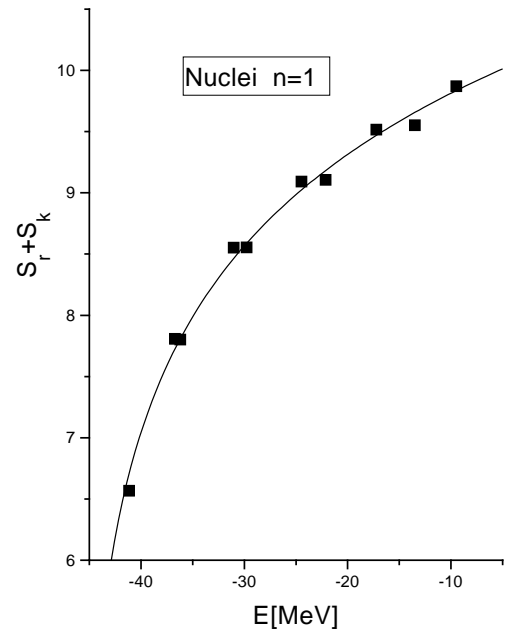

FIG. 5. The same as in Fig. 4 but for a proton in $\mathrm{Pb}^{208}$ according to the Sk III interaction for $n=1$.

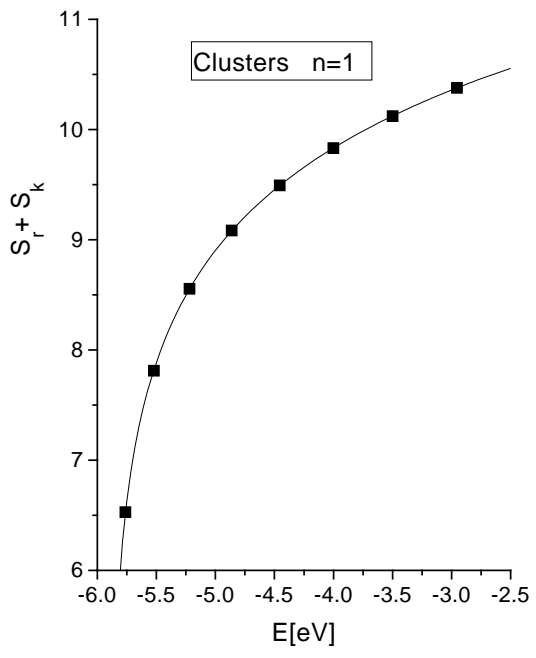

FIG. 6. The same as in Fig. 4 but for an electron in atomic clusters $(\mathrm{N}=198)$ using the Woods-Saxon potential for $n=1$. 
TABLE I. Values of the parameters $a$ and $b$ which appear in the expressions $S_{r}$ (or $\left.S_{k}\right)=a+b N^{1 / 3}$ for a nucleon (proton) in nuclei according to the Sk III interaction, a $\Lambda$ in hypernuclei according to a relativistic model and an electron in atomic clusters with Woods-Saxon potential.

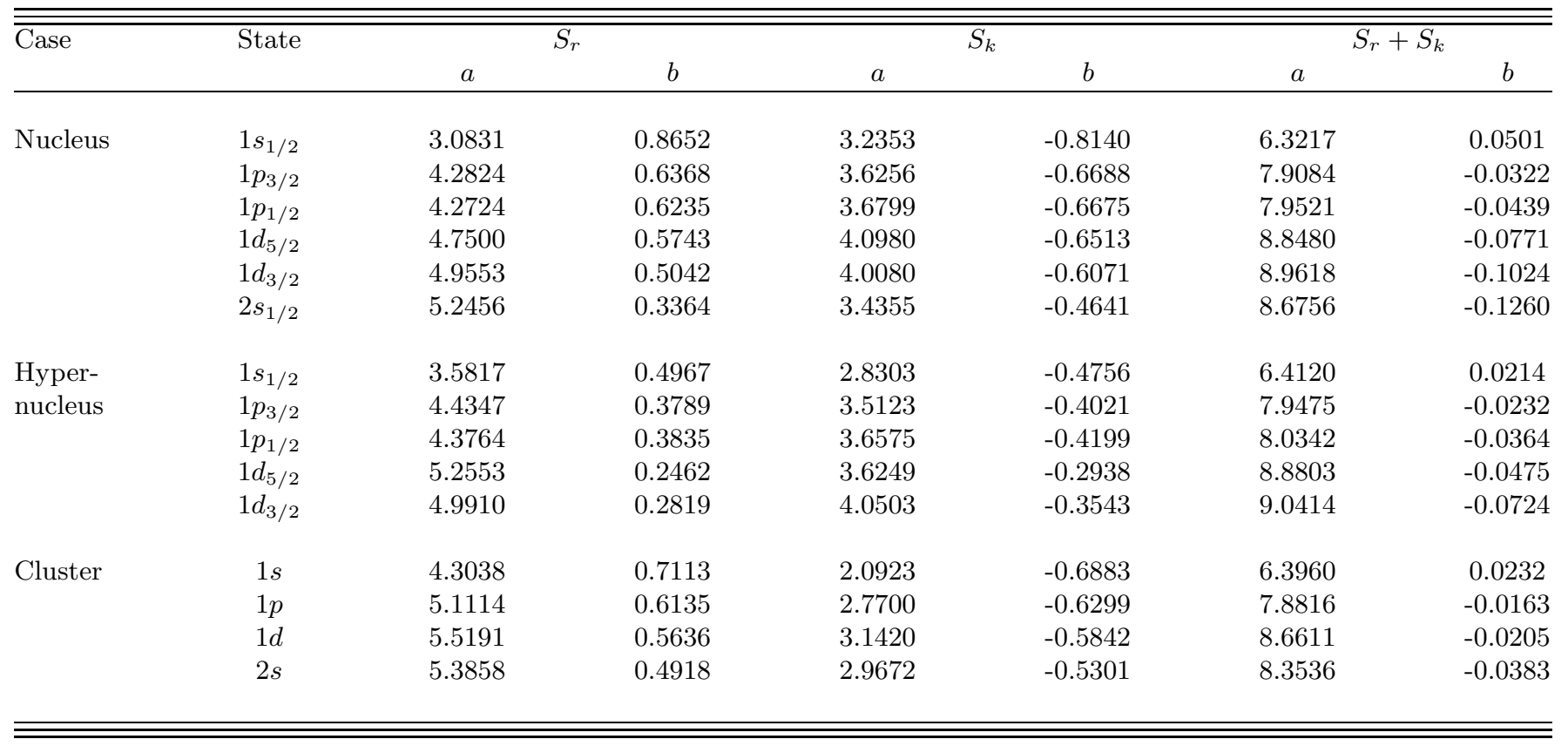

